\title{
The Mitotic Chromosomes of the Cellular Slime Mould Dictyostelium discoideum: A Karyotype Based on Giemsa Banding
}

\author{
By GILLIAN E. ROBSON AND KEITH L. WILLIAMS \\ Department of Genetics, Research School of Biological Sciences, \\ The Australian National University, P.O. Box 475, Canberra City 2601, \\ A.C.T., Australia
}

(Received 2 July 1976; revised 26 September 1976)

\begin{abstract}
SUMMARY
All seven mitotic chromosomes of the haploid cellular slime mould Dictyostelium discoideum have been identified and numbered using Giemsa banding techniques. Although size differences are not marked, the size distribution is one long, three medium length, and three shorter chromosomes. Each chromosome has a distinctive banding pattern. All seven chromosomes appear to have terminal or near terminal centromeres. Apparent connexions between chromosomes explain the controversy concerning chromosome number (five or seven) in $D$. discoideum. The mitotic index of exponential-phase axenic cultures of $D$. discoideum is $2 \%$. By returning aggregating amoebae to growth medium the mitotic index could be reproducibly raised to approximately $6 \%$.
\end{abstract}

\section{INTRODUCTION}

In general the examination of mitotic chromosomes in simple eukaryotes by light microscopy has been unsatisfactory because of their small size and incomplete condensation during mitosis, e.g. yeast (Wintersberger, Binder \& Fischer, 1975). By contrast the chromosomes of the simple eukaryote Dictyostelium discoideum Raper have proved amenable to microscopic examination, despite the fact that $D$. discoideum has a DNA content only approximately I I times that of Escherichia coli, so that on average each chromosome has approximately $\mathrm{I} \cdot 5 \times$ an $E$. coli genome (Jacobson \& Lodish, 1975). Although excellent mitotic preparations of $D$. discoideum have been reported using aceto-orcein (Wilson, 1953; Ross, 1960; Sussman, 196I), the introduction of air-drying techniques and Giemsa stain has made the examination of the chromosomes much easier (Brody \& Williams, I974). Studies by Wilson (1953), Ross (I960), Sussman (I96I), and Brody \& Williams (I974) all showed that the haploid chromosome number of $D$. discoideum is seven. Recently a karyotype of $D$. discoideum has been proposed in which the chromosome number is said to be five rather than seven (Muroyama et al., I975); this revives an earlier controversy in which it was argued that $D$. discoideum may have less than seven chromosomes (see Bonner, 1967). In this report we present a karyotype based on Giemsa banding techniques which leaves no doubt that the haploid chromosome number is seven.

\section{METHODS}

Culture of amoebae of $D$. discoideum. Amoebae of strain AX3 (Loomis, 197I) were grown in $70 \mathrm{ml}$ axenic medium (Watts \& Ashworth, 1970) in $250 \mathrm{ml}$ flasks in an orbital shaker ( 150 cycles $\mathrm{min}^{-1}$ ) at $22{ }^{\circ} \mathrm{C}$. Amoebae were inoculated at $10^{4}$ to $10^{5} \mathrm{ml}^{-1}$, the doubling time was approximately $\mathrm{Io} \mathrm{h}$, and the stationary phase was $2 \times 10^{7}$ amoebae $\mathrm{ml}^{-1}$. 
Increasing the mitotic index. Amoebae were aggregated on filters in a manner similar to that described by Sussman (I966). Amoebae approaching the stationary phase (about $7 \times 10^{6} \mathrm{ml}^{-1}$ ) were harvested from axenic medium by centrifugation (Sorvall SS-34 head; I $500 \mathrm{rev} . \mathrm{min}^{-1} ; 2 \mathrm{~min}$ ) and washed three times in salt solution [containing $\left(\mathrm{g} \mathrm{l}^{-1}\right): \mathrm{NaCl}$, $\left.0.6 ; \mathrm{KCl}, 0.75 ; \mathrm{CaCl}_{2}, 0.3\right]$ using the same centrifugation. The final amoebal density was adjusted to $10^{8} \mathrm{ml}^{-1}$. Amoebae $\left(10^{8}\right)$ were pipetted on to a $7 \mathrm{~cm}$ diam. Whatman no. 50 filter resting on top of a Whatman no. 17 filter moistened with LPS solution [containing $\left(\mathrm{g} \mathrm{l}^{-1}\right): \mathrm{KCl}, \mathrm{I} \cdot 5 ; \mathrm{MgCl}_{2}, 0.5 ; \mathrm{KH}_{2} \mathrm{PO}_{4}, 5.45$; dihydrostreptomycin sulphate, $0.5 ; \mathrm{pH} \mathrm{6.5]}$. The filters were incubated in moist conditions for 7 to $9 \mathrm{~h}$, and then the aggregating amoebae were gently washed from the filter with axenic medium, separated by vortexing and counted. Several fresh axenic cultures (see above) were established immediately by adding amoebae to $70 \mathrm{ml}$ axenic medium, so that the final concentration was between $2.5 \times 10^{5}$ and $5 \times 10^{5} \mathrm{ml}^{-1}$. At zero time and subsequently, samples were removed, fixed and stained. When the mitotic index was above $4 \%$ a large number of air-dried slides were prepared for subsequent banding treatments.

Fixation procedure. In all experiments amoebae were fixed in methanol/acetic acid (3:I, $\mathrm{v} / \mathrm{v}$ ) and air-dried on to clean slides as described previously (Brody \& Williams, 1974).

Staining. The standard procedure involved staining air-dried slides in $10 \%(\mathrm{v} / \mathrm{v})$ Gurr's Giemsa stain (Improved R66) in 0.07 M-Sorensen's phosphate pH 6.8 for 10 min.

G-banding treatments. The following prestaining treatments of air-dried slides were tested: $2 \times \mathrm{SSC}\left(0.3 \mathrm{M}-\mathrm{NaCl} / 0.03 \mathrm{M}\right.$-trisodium citrate) at 60 to $75{ }^{\circ} \mathrm{C}$ for between 10 and $90 \mathrm{~min} ; 0.25 \%(\mathrm{w} / \mathrm{v})$ trypsin (Difco, $\mathrm{I}: 250$ ) in $0.85 \%(\mathrm{w} / \mathrm{v}) \mathrm{NaCl}$ for between 30 and $90 \mathrm{~s}$ at room temperature $\left(2 \mathrm{I}{ }^{\circ} \mathrm{C}\right.$ ); BSSR (Hanks' balanced salt solution without $\mathrm{Ca}^{2+}$ and $\mathrm{Mg}^{2+}$ ) for between 2 and $3 \mathrm{~min}$ at room temperature; $0.025 \%(\mathrm{w} / \mathrm{v})$ EDTA and $0.25 \%(\mathrm{w} / \mathrm{v})$ trypsin in BSSR for between 30 and $\mathrm{I} 20 \mathrm{~s}$ at room temperature (Dev, Warburton \& Miller, I972; Hsu, 1973). Staining with Giemsa at $\mathrm{pH} 90^{\circ}$ instead of $\mathrm{pH} 6.8$ was also tried (Patil, Merrick \& Lubs, 1971).

$C$-banding treatments. The treatment using saturated barium hydroxide in the range 20 to $65{ }^{\circ} \mathrm{C}$ for up to Io min, followed immediately by $2 \times \mathrm{SSC}$ at 50 to $75{ }^{\circ} \mathrm{C}$ for $30 \mathrm{~min}$ was used (Weimarck, 1975).

Determination of the mitotic index. The percentage of mitotic nuclei was determined by examining all nuclei in a series of microscope fields chosen at random using $1000 \times$ magnification. Between 600 and 1000 nuclei were examined for each sample. To remove cytoplasmic staining and hence emphasize the staining of interphase nuclei, slides were routinely treated with $2 \times \mathrm{SSC}$ at $60^{\circ} \mathrm{C}$ for $30 \mathrm{~min}$, although slides not treated in this way gave the same mitotic indices. Trypsin treatment also made interphase nuclei more prominent by decreasing cytoplasmic staining.

Microscopyand photographic techniques. Slides were examined under $1000 \times$ or $1600 \times$ magnification, using a Carl Zeiss photomicroscope IIl, with bright-field green illumination. In some cases phase optics were used. Kodak Panatomic $X$ film was used and the most favourable contrast was achieved on Agfa grade 5 or grade 6 print paper by developing in Kodak DA-r63 (dilution $I+3$ ) for 2 to $2 \cdot 5$ min with agitation (Davidson, I973).

\section{RESULTS}

\section{Increasing the mitotic index}

Although accurate estimation of the mitotic index was not simple because mitoses were not randomly distributed, the mitotic index, based on the examination of rooo nuclei, was 
Table I. Distribution of numbers of mitotic nuclei in randomly chosen microscope fields

\begin{tabular}{|c|c|c|c|c|c|c|c|c|c|c|c|}
\hline \multirow{2}{*}{\multicolumn{2}{|c|}{ Expt $\dagger$}} & \multirow[b]{2}{*}{$n$} & \multirow[b]{2}{*}{$\ldots$} & \multicolumn{8}{|c|}{ No. of fields of view having $n$ mitoses* } \\
\hline & & & & \multirow{2}{*}{$\begin{array}{c}0 \\
236 \\
209\end{array}$} & \multirow{2}{*}{$\begin{array}{c}1 \\
48 \\
86\end{array}$} & \multirow{3}{*}{$\begin{array}{c}2 \\
23 \\
18\end{array}$} & \multirow{2}{*}{$\begin{array}{r}3 \\
3 \\
\end{array}$} & \multirow{2}{*}{$\begin{array}{l}4 \\
3\end{array}$} & \multirow{2}{*}{$\begin{array}{l}5 \\
3\end{array}$} & \multirow{2}{*}{$\begin{array}{l}6 \\
0\end{array}$} & \multirow{2}{*}{$\begin{array}{l}7 \\
0\end{array}$} \\
\hline I & Obs. & & & & & & & & & & \\
\hline & Exp. & & & & & & & & 2 & & \\
\hline \multirow[t]{2}{*}{2} & Obs. & & & 234 & 48 & 13 & 6 & $\mathbf{I}$ & 0 & 0 & I \\
\hline & Exp. & & & 216 & 73 & 12 & & & I & & \\
\hline
\end{tabular}

* The mean number of nuclei per field of view was 20 (S.D. 12, range $\circ$ to 65 nuclei) for expt 1 , and 23 (s.D. I4, range o to 82 nuclei) for expt 2; magnification was $\times 1000$.

$\dagger$ Obs., Observed values; Exp., expected values, assuming a Poisson distribution of mitotic nuclei. Expt I, $\chi^{2}=46 \cdot 2$, d.f. $=2$; expt $2, \chi^{2}=59^{\circ}$ I, d.f. $=2$ (Snedecor, 1961). It was assumed that the variation in the number of nuclei per field would not interfere with these calculations.

$2 \%$ for exponential-phase axenic cultures. Table $\mathrm{I}$ shows the incidence of mitotic figures in a large number of random microscope fields in two experiments. In about one quarter of the fields, one or more mitotic figures were observed. In two thirds of these fields, only one mitosis was observed, while in the remaining fields usually two, but as many as seven mitoses were observed per field. Chi-square tests confirm that the mitotic nuclei are not distributed at random. There were less single mitoses and more multiple mitoses than would be predicted from a Poisson distribution (Table I). It appears likely that many of these clustered mitoses represent multinucleate cells. Because the air-drying technique spreads the cells and breaks the plasma membrane, it was difficult to be certain of this. However, on the rare occasions when poor spreading occurred due to unclean slides, multinucleate cells were observed (Fig. $4 f$ ). When the mitotic index was calculated, each mitosis was counted (e.g. a tetranucleate cell was counted as four), since we presume that interphase cells show the same spectrum of single to multiple nuclei as that found in dividing cells (see Fig. $4 f$ ).

Aggregation of amoebae on filters for $7.5 \mathrm{~h}$ followed by return to axenic medium resulted in a lag during which the mitotic index was very low for about 6 to $6.5 \mathrm{~h}$ (Fig. $\mathrm{I} b$, open bars). This was followed by a sharp and reproducible increase in mitotic index to $6 \%$. After $7.5 \mathrm{~h}$ on filters, strain $\mathrm{Ax} 3$ formed small mounds of amoebae which became tighter by $9 \mathrm{~h}$. Amoebae aggregated for $9 \mathrm{~h}$ produced similar results to those found for amoebae aggregated for $7.5 \mathrm{~h}$, although the maximum mitotic index achieved was slightly lower (Fig. I $b$, closed bars). Although it was not the aim of this study to demonstrate synchrony of $D$. discoideum, the results in Fig. I suggest that some synchrony in mitosis occurred not only at the initial division but also at subsequent divisions. Studied over the same period, the mitotic index of the control cultures remained approximately constant (Fig. I $a$ ). Both 'synchronized' and control cultures showed the same ratio of uninucleate to multinucleate amoebae.

An increased mitotic index was also observed when stationary-phase amoebae were returned to fresh growth medium, although we have not examined this method in detail.

\section{Giemsa banding treatments}

Both C- and G-banding treatments were tested. As is commonly observed, similar treatments on different days did not always produce the same result. Some untreated preparations showed some banding. 

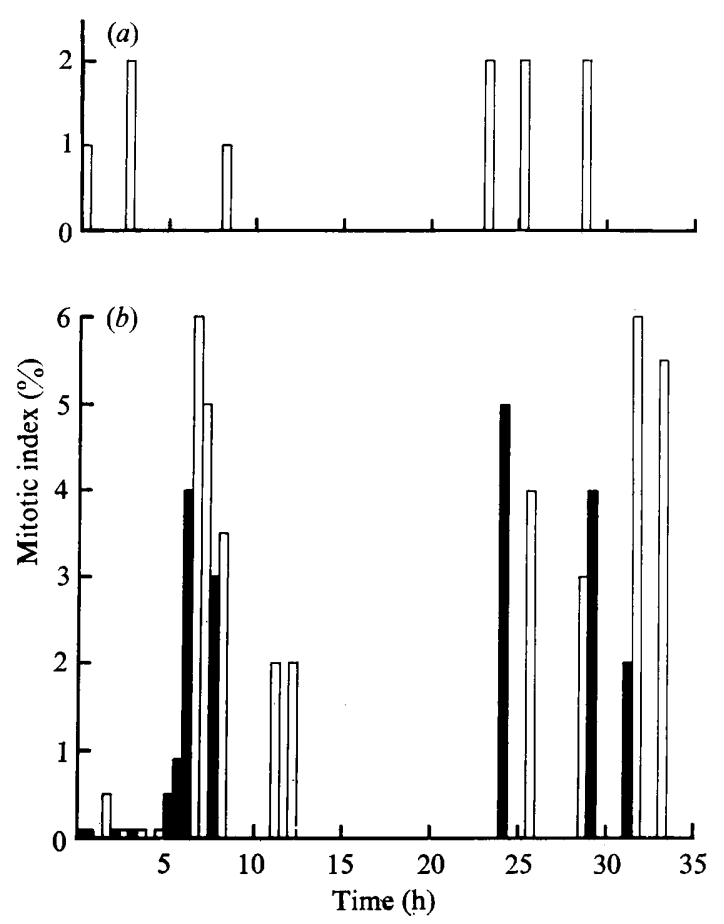

Fig. I. Mitotic index of $(a)$ control and $(b)$ 'synchronized' axenic cultures of $D$. discoideum at various times after the establishment of a culture in axenic medium. For the control, zero time refers to an exponential-phase culture at $2.5 \times 10^{5}$ amoebae $\mathrm{ml}^{-1}$. The zero time in 'synchronized' amoebae represents the time when aggregating amoebae were removed from filters and returned to axenic medium at a density of about $2.5 \times 10^{5}$ amoebae $\mathrm{ml}^{-1}$. Two experiments are shown for 'synchronized' amoebae after aggregation on filters for $7.5 \mathrm{~h}$ (open bars) and $9 \mathrm{~h}$ (closed bars) at $22{ }^{\circ} \mathrm{C}$ before being returned to axenic medium. Haemocytometer counts indicated that the number of amoebae had doubled by 7 to Io $h$ after zero time, and subsequently the doubling time was 7 to $10 \mathrm{~h}$.

$G$-banding. In general neither use of Giemsa stain at $\mathrm{pH} 9$, nor incubation in $2 \times \mathrm{SSC}$ over a range of temperatures (60 to $75^{\circ} \mathrm{C}$ ) for a range of times (Io to $90 \mathrm{~min}$ ) before Giemsa staining, produced consistent banding. In some cases use of BSSR for $2 \mathrm{~min}$ at room temperature before staining gave successful banding, as did a combination of trypsin, BSSR and EDTA (Fig. 2b). By far the most successful and consistent G-banding technique involved trypsin treatment before Giemsa staining. The best conditions were $0.25 \%(\mathrm{w} / \mathrm{v})$ trypsin for $60 \mathrm{~s}$ at room temperature, followed by the standard staining procedure. Trypsin treatment for less than $60 \mathrm{~s}$ resulted in progressively less banding, whereas treatment for more than $60 \mathrm{~s}$ was destructive to the chromosomes.

$C$-banding. Some difficulty was experienced with C-banding treatments in striking a balance between too much destruction and incomplete effect. Although really satisfactory preparations have not been achieved, treatment with freshly prepared $5 \%(\mathrm{w} / \mathrm{v}) \mathrm{Ba}(\mathrm{OH})_{2}$ for 10 min at 45 to $50{ }^{\circ} \mathrm{C}$ followed immediately by incubation in $2 \times \mathrm{SSC}$ at $65^{\circ} \mathrm{C}$ for $30 \mathrm{~min}$, seems promising. Sometimes treatment with trypsin gave preparations which looked more like C-than G-banding (Fig. 2a). 


\section{The karyotype}

The characteristic banding pattern and manner of chromatid separation were the most useful features for identifying the chromosomes (Fig. 2). Difference in chromosome length was also helpful, but could not always be relied on since in late prophase and even early metaphase the differences were small (Fig. $4 b$ and $c$ ). Although early prophase chromosomes were considerably longer and had more bands, the problems of irregular rates of contraction and chromosomal connexions (see below) made it much easier to identify the karyotype using late prophase and early metaphase chromosomes (Fig. 2). A difficulty encountered previously (Brody \& Williams, 1974) was that some prophases contained a short chromosome while others did not. This is most probably explained by differential rates of condensation; chromosome 5, in particular, was observed to condense sooner than the other six chromosomes in several prophases (Fig. 4a).

Our studies on anaphase preparations indicate that all seven chromosomes have terminal or near terminal centromeres. This is confirmed by the electron-microscope studies of Moens (1976) which show clear terminally located kinetochore plates on all seven chromosomes (Figs $3 a$ and $4 h$, Moens, 1976). This view is also supported by the fact that in our late prophase and early metaphase preparations, sister chromatids of chromosomes $\mathrm{I}, 2,3,4$ and 5 have been seen to be joined at one end only; sister chromatid separation is less well established in the smaller chromosomes 6 and 7, although they too appear to have terminal centromeres. Representative metaphases showing banded chromosomes with or without separated chromatids, are presented in Fig. 2.

Chromosome $\mathrm{I}$. This is the longest chromosome. It is usually bent close to the centromere and again about two-thirds distal to the centromere, giving it a 'squiggly' appearance. In banded preparations up to five bands uniformly spaced along its length are observed Figs $2 b$ and 3 ). The terminal and subterminal bands at the centromeric end and the subterminal band at the end distal to the centromere usually stain most intensely.

Chromosome 2. This is slightly shorter than chromosome I and similar in size to chromosomes 3 and 4 . When chromatids are observed they are commonly crossed distal to the centromere (Fig. 2a). Banded preparations usually show three major bands, two of which (situated at or near either end of the chromosome) are prominent, while the third, midway between them, is more lightly stained (Fig. 3). It is important to note that while the band distal to the centromere is distinctly terminal, the band at the centromeric end is not.

Chromosome 3. This is most easily recognized by the chromatids flaring from a point about two-thirds distal to the centromeric end. This partial chromatid separation is often observed before chromatid separation in the other long chromosomes (Fig. 2b). In banded preparations four bands are observed (Fig. 3). The two bands close to the centromere may fuse to form a heavily stained bar. The other two bands are less distinct and are located at the centre and the end of the chromosome distal to the centromere.

Chromosome 4. At first glance this chromosome can be confused with chromosome 2. Typically the chromatids separate initially at the centre of the chromosome and form a distinctive hollow lens shape (Fig. 2a). Banded preparations show two heavily stained regions at either end of the chromosome (Fig. 3). This clearly distinguishes it from chromosome 2 which has only one terminal band (Fig. $2 b$ ). There is a third band that is less heavily stained situated close to the centromeric end (Fig. 3).

Chromosome 5. This is generally the first to condense and sometimes does so well in advance of the others (Fig. 4 a). It is usually the first chromosome to show chromatid separation. It is one of the three shorter chromosomes and is easily recognized by the heavy bands at each end (Figs $2 a, b$ and 3 ). 
(a)

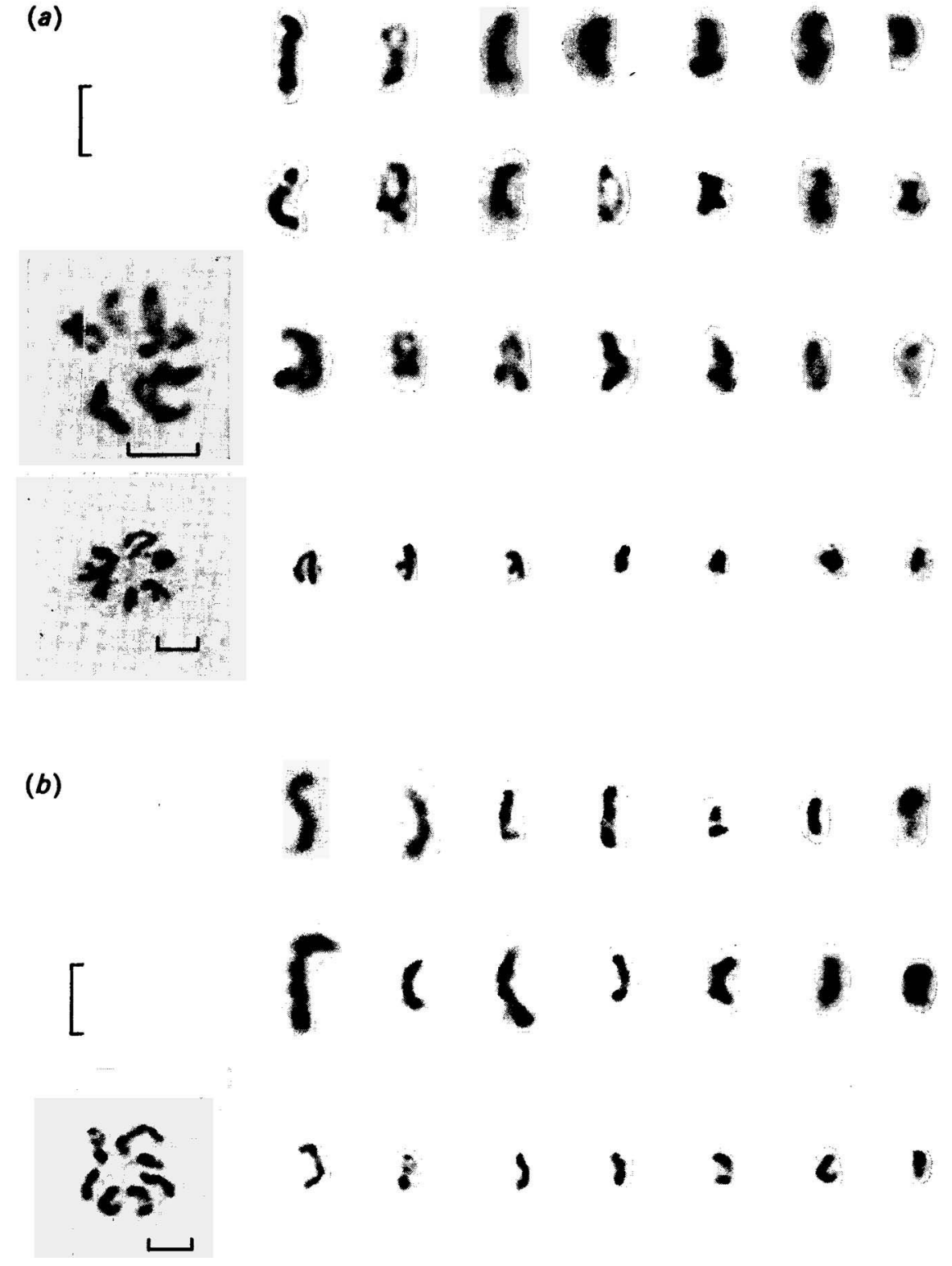

Fig. 2. Karyotype of banded chromosomes of $D$. discoideum showing chromosomes with (a) separated and $(b)$ unseparated chromatids. For both $(a)$ and $(b)$ two well banded examples of each chromosome are presented; these were obtained from several different cells. In addition, two examples $(a)$ and one example $(b)$ of cells with all chromosomes identifiable are shown. The banding treatments were $0.25 \%(\mathrm{w} / \mathrm{v})$ trypsin in $0.85 \%(\mathrm{w} / \mathrm{v}) \mathrm{NaCl}$ for $70 \mathrm{~s}$ and $60 \mathrm{~s}$ respectively for the two cells in $(a)$, and $0.25 \%(\mathrm{w} / \mathrm{v})$ trypsin plus $0.025 \%(\mathrm{w} / \mathrm{v})$ EDTA in BSSR for $60 \mathrm{~s}$ for the cell in (b). The chromosomes are oriented such that the centromere is uppermost. Bar markers represent $2 \mu \mathrm{m}$. 


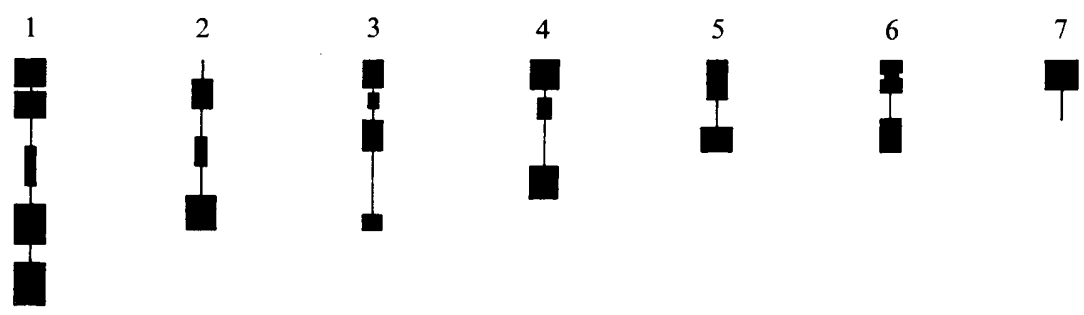

Fig. 3. Proposed karyotype of haploid $D$. discoideum based on trypsin-banded chromosomes. In each case the centromere probably lies at the top of the chromosome. Because of the different rates of contraction of the chromosomes the relative lengths represent a notional guide only.

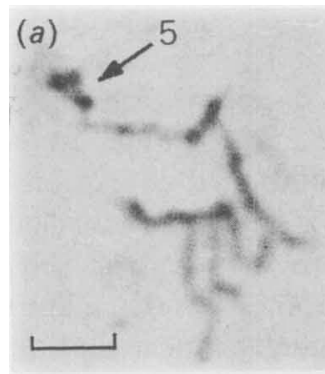

(c)

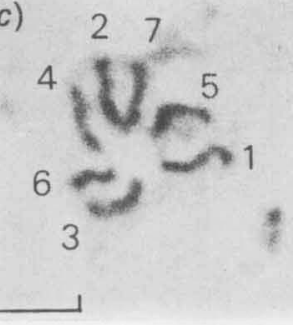

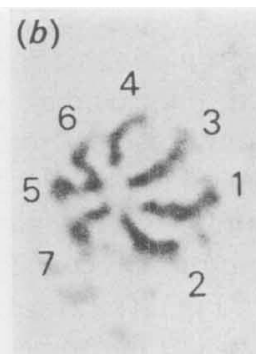

(d)
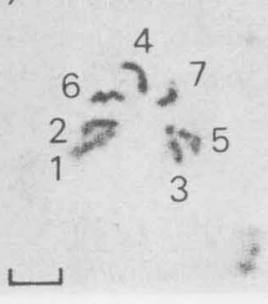

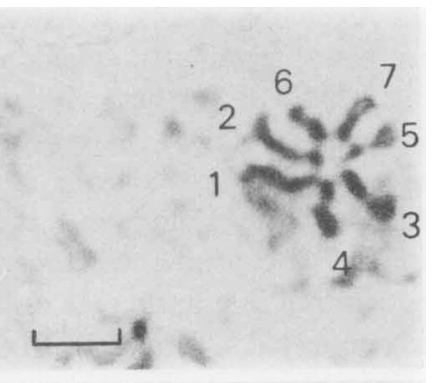

(e)

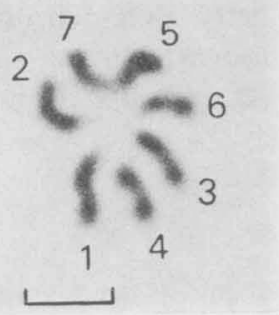

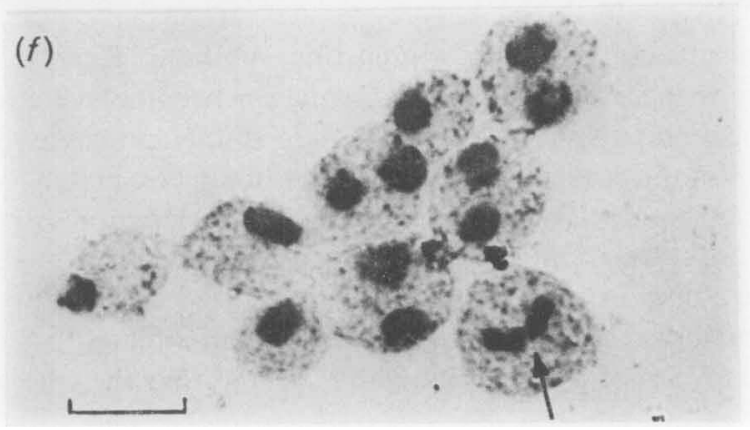

Fig. 4. (a) Early condensation of chromosome 5 (arrowed). $(b-e)$ Chromosomal connexions, with individual chromosomes numbered: $(b)$ two metaphases with chromosomes connected in a ring; $(c, d, e)$ connexions between pairs of chromosomes at metaphase. $(f)$ Multinucleate cells; the arrow shows two synchronized telophases in a binucleate cell. The cells were not disrupted in $(f)$ because the slide was unclean. All preparations were treated with trypsin. Bar markers represent $2 \mu \mathrm{m}$ for $(a-e)$, and $10 \mu \mathrm{m}$ for $(f)$. 
Chromosome 6. This is the most variable in appearance but is commonly recognizable by its shallow S-shape (Fig. 2). The position of the centromere is not clear, although there is some indication that it is terminal (Fig. $2 a$ ). Banded preparations usually show either two or three bands (Fig. 3).

Chromosome 7. This is the shortest of the chromosomes at metaphase (Fig. $2 b$ ) but is quite long before condensation (Fig. $4 b$ and $c$ ). In banded preparations, when fully condensed, it is easily recognizable as a darkly stained spot with a pale tail (Fig. 3). Chromatid separation is observed but the small size makes positioning of the centromere difficult, although it is most probably terminal (Fig. 2a).

\section{Chromosomal connexions}

It is often difficult to count the chromosomes in early prophase as they appear to be connected. As condensation progresses and they become arranged in a radial fashion on the metaphase plate, terminal connexions are sometimes apparent between adjacent chromosomes (Fig. $4 b$ ). With further condensation, these connexions disappear. This process most probably occurs in stages, as sometimes only pairs of joined chromosomes are observed. Figure $4(c, d$ and $e)$ shows three metaphases in which chromosomal connexions are apparent. Although the banding in Fig. $4(c$ and $e)$ is incomplete, we attempted to identify each chromosome to determine whether chromosomal connexions were specific and whether breakage occurred in a particular order. In Fig. 4(c), chromosomes 6 and 4,2 and 7 , and 5 and $\mathrm{I}$ are connected, whereas in Fig. $4(d)$, chromosomes 5 and 3 , and $\mathrm{I}$ and 2 are connected, and in Fig. $4(e)$, chromosomes 5 and 7 are connected. It appears therefore that there is no specificity in these chromosomal connexions. If the identity of each of the chromosomes had not been established, a possible interpretation would have been that the metaphases in Fig. $4(c, d$ and $e$ ) had not seven chromosomes but four, five and six chromosomes respectively.

\section{DISCUSSION}

The low mitotic index of exponential-phase axenic cultures of $D$. discoideum made it tedious to attempt to establish the karyotype. As yet we have not found conditions for metaphase arrest using colchicine or vinblastine sulphate. Katz \& Bourguignon (1974) reported that aggregating amoebae of $D$. discoideum become synchronized. We reasoned that, by returning aggregating amoebae to growth medium, an increased mitotic index might be observed; such was the case and mitotic indices of approximately $6 \%$ made the work of identifying the chromosomes much easier. Katz \& Bourguignon (1974) suggested in their study that mitosis occupies about $15 \%$ of the cell cycle. Their estimates were based on the observed step of doubling of cell number in synchronized cultures and seem liable to considerable error. Our finding that mitosis represents approximately $2 \%$ of the cell cycle time is supported by the results of Ross (1960) who observed that the whole process of rounding up of a bacterially grown amoeba of $D$. discoideum to the separation of the two daughter amoebae took between 3 and Io min out of a cell cycle time of the order of $4 \mathrm{~h}$.

In two previous studies (Wilson, 1953; Ross, 1960) photographs have been published showing what we believe to be sister chromatids, partially separated both in late prophase and early metaphase. These reports were published when little was known of the nuclear behaviour of $D$. discoideum, and the photographs were interpreted as showing paired homologues at meiosis. Although the chromosomes showing chromatid twisting and separation (Fig. 2a) superficially resemble meiotic preparations of higher organisms, such a resemblance 
is clearly misleading. Only haploid amoebae dividing by binary fission were used in this study. There is genetic evidence that in two closely related species of slime moulds (Dictyostelium mucoroides and Dictyostelium giganteum) meiosis occurs in a specific sexual structure, the macrocyst. The macrocyst is almost certainly the sexual structure in D. discoideum too (Jacobson \& Lodish, 1975). Synaptonemal complexes, which are characteristic of meiosis, have been observed in macrocysts of the cellular slime mould Polysphondylium violaceum (Erdos, Nickerson \& Raper, 1972). The characteristic and easily observed separation of the sister chromatids seems to occur at a specific stage in mitosis, between late prophase and early metaphase, in all cells. At present there is no accepted explanation for this phenomenon although it may depend on the small size of the chromosomes concerned. Attempts are being made to examine meiotic chromosomes in macrocysts of $D$. discoideum to compare their morphology with the mitotic chromosomes observed here.

Apparent chromosomal connexions are common in $D$. discoideum. Often in early prophase it is difficult to distinguish chromosomes which appear to be associated end to end. Such connexions have been reported in a number of systems (Du Praw, 1970). Whether these chromosomal connexions reflect a higher level of nuclear organization, such as has been suggested in yeast (Lauer \& Klotz, 1975; Wintersberger et al., 1975), remains to be established. It seems that if there are connexions, they break up after the chromosomes become arranged on the metaphase plate. In most cases seven individually recognizable chromosomes are observed at metaphase (Fig. 2). When fewer than seven chromosomes are observed, invariably this is caused by ambiguous overlap of chromosomes, or association between two chromosomes which can be recognized as individuals using banding techniques (Fig. $4 c, d, e$ ). Hence we conclude that $D$. discoideum has seven chromosomes. We believe that the recent suggestion by Muroyama et al. (1975) that $D$. discoideum has only five chromosomes failed to consider chromosomal connexions.

Evidence from two other sources confirms that haploid $D$. discoideum has seven chromosomes. Firstly, a recent electron-microscope study of mitosis in haploid D. discoideum demonstrated seven pairs of kinetochores (Moens, 1976). Secondly, although mitotic genetic analysis in $D$. discoideum is only beginning (Jacobson \& Lodish, 1975), at least six, and probably all seven mitotic linkage groups have been established (Williams, unpublished). In other systems the number of mitotic linkage groups gives an accurate estimate of the chromosome number (Kafer, I 958). The identification of the chromosomes with banding techniques described here opens the way to correlating the genetic and cytological maps.

Note added in proof. Zada-Hames [Journal of General Microbiology (1977) 99, 20I-208], in an independent study in which slightly different techniques were used, obtained almost identical banding patterns for the chromosomes. A standard numbering system has been agreed upon and is used in both papers. In her paper, Zada-Hames has conclusively shown that $D$. discoideum strain $\mathrm{AX} 2$ is a normal haploid strain and that it is not highly aneuploid. Muroyama et al. (1975) claimed that, while $D$. discoideum strain NC4 is a stable haploid, strains $\mathrm{M}_{3}$ and $\mathrm{AX} 2$ showed variable chromosome numbers. $\mathrm{Dr} \mathrm{T}$. Muroyama kindly sent us his strains NC4, AX2 and M3. A preliminary examination of them suggests that his strain $\mathrm{NC}_{4}$ is haploid, but strains AX2 and $\mathrm{M} 3$ from his laboratory are diploid. Hence, variable chromosome numbers would be expected in the strains M3 and AX2 of Muroyama et al. (1975), as has been shown in a previous study on diploid strains of $D$. discoideum (Brody \& Williams, 1974.)

We thank Dr Graham Webb, Dr Barry Rolfe and Professor Bernard John for helpful comments. 


\section{REFERENCES}

Bonner, J. T. (1967). The Cellular Slime Molds, 2nd edn. Princeton, New Jersey, U.S.A.: Princeton University Press.

Brody, T. \& Williams, K. L. (1974). Cytological analysis of the parasexual cycle in Dictyostelium discoideum. Journal of General Microbiology 82, 371-383.

Davidson, N. R. (1973). Photographic techniques for recording chromosome banding patterns. Journal of Medical Genetics 10, 122-126.

Dev, V. G., Warburton, D. \& Miller, O. J. (1972). Giemsa banding of chromosomes. Lancet i, 1285.

Du PrAW, E. (1970). DNA and Chromosomes. New York: Holt, Reinhart and Winston.

ERdos, G. W., Nickerson, A. W. \& RAPER, K. B. (1972). Fine structure of macrocysts in Polysphondylium violaceum. Cytobiologie 6, 35I-366.

Hsu, T. C. (1973). Longitudinal differentiation of chromosomes. Annual Review of Genetics 7, I53-176.

JACOBson, A. \& Lodish, H. F. (1975). Genetic control of development of the cellular slime mold, Dictyostelium discoideum. Annual Review of Genetics 9, 145-185.

KAFER, E. (1958). An eight chromosome map of Aspergillus nidulans. Advances in Genetics 9, 105-I45.

KATZ, E. R. \& Bourguignon, L. Y. W. (1974). The cell cycle and its relationship to aggregation in the cellular slime mold, Dictyostelium discoideum. Developmental Biology 36, 82-87.

LAUER, G. D. \& KLOTZ, L. C. (1975). Determination of the molecular weight of Saccharomyces cerevisiae nuclear DNA. Journal of Molecular Biology 95, 327-330.

Loomis, W. F. (197I). Sensitivity of Dictyostelium discoideum to nucleic acid analogues. Experimental Cell Research 64, 484-486.

MoENs, P. B. (1976). Spindle and kinetochore morphology of Dictyostelium discoideum. Journal of Cell Biology 68, $113-122$.

Muroyama, T., Hashimoto, Y., Sameshima, M. \& Yamada, T. (1975). The karyotype of the cellular slime molds. Chromosome Information Service 19, 32-33.

Patil, S. R., MerRick, S. \& LUBS, H. A. (197I). Identification of each human chromosome with a modified giemsa stain. Science 173, $82 \mathrm{I}-822$.

Ross, I. K. (1960). Studies on diploid strains of Dictyostelium discoideum. American Journal of Botany 47, 54-59.

SNedeCoR, G. W. (196I). Statistical Methods, 5th edn. Iowa, U.S.A.: Iowa State University Press.

Sussman, M. (I966). Biochemical and genetic methods in the study of cellular slime mold development. Methods in Cell Physiology, vol. 2, pp. 397-4 Io. Edited by D. Prescott. New York: Academic Press.

Sussman, R. R. (196I). A method for staining the chromosomes of Dictyostelium discoideum myxamoebae in the vegetative stage. Experimental Cell Research 24, 154-155.

WATts, D. J. \& Ashworth, J. M. (1970). Growth of myxamoebae of the cellular slime mould Dictyostelium discoideum in axenic culture. Biochemical Journal $119,17 \mathrm{I}-174$.

WeIMARCK, A. (1975). Heterochromatin polymorphism in the rye karyotype as detected by the Giemsa C-banding technique. Hereditas 79, 293-300.

Wilson, C. M. (1953). Cytological study of the life cycle of Dictyostelium. American Journal of Botany 40, 714-718.

Wintersberger, U., Binder, M. \& Fischer, P. (1975). Cytogenetic demonstration of mitotic chromosomes in yeast Saccharomyces cerevisiae. Molecular and General Genetics 142, $13-17$. 